\title{
Application-based Smart Parking System using CAN Bus
}

\author{
Rahul M Patil, N R Vinay, Pratiba D \\ Department of Computer Science and Engineering R.V. College of Engineering Bangalore-560059, \\ Karnataka, India
}

\begin{tabular}{|c|c|}
\hline Article Info & ABSTRACT \\
\hline $\begin{array}{l}\text { Article history: } \\
\text { Received May 25, } 2018 \\
\text { Revised Jul 26, } 2018 \\
\text { Accepted Aug 9, } 2018\end{array}$ & $\begin{array}{l}\text { We have witnessed a rapid growth in the Internet of Things and its } \\
\text { contribution to the growth of Smart cities in recent years. Consistent efforts } \\
\text { have been undertaken by the Government of India to elevate the growth of } \\
\text { this industry. The increase in population and vehicular densities have led to } \\
\text { congested roads, inadequate parking facilities and poor infrastructure has } \\
\text { called for a technology-driven solution known com-monly as a Smart }\end{array}$ \\
\hline $\begin{array}{l}\text { Keywords: } \\
\text { Android } \\
\text { Arduino } \\
\text { CAN } \\
\text { Cloud database } \\
\text { Internet of things } \\
\text { Smart parking }\end{array}$ & $\begin{array}{l}\text { Parking System (SPS). The solution proposed in this paper is a client-based } \\
\text { parking slot reservation system that is implemented using micro-controllers } \\
\text { interfaced with sensors for parking vacancy detection. They are also } \\
\text { connected to cloud services in order to produce a complete IoT solution, with } \\
\text { each micro-controller acting as a node in a network. Additionally, the CAN } \\
\text { protocol is deployed for communication between nodes of this network in } \\
\text { case of failure of the nodes in the wireless network. This is a solution de- } \\
\text { veloped that caters to users mainly residing in Smart Cities and addresses the } \\
\text { issue of network node failure. }\end{array}$ \\
\hline & $\begin{array}{r}\text { Copyright }\left({ }^{\circ} 2018 \text { Institute of Advanced Engineering and Science. }\right. \\
\text { All rights reserved. }\end{array}$ \\
\hline \multicolumn{2}{|l|}{ Corresponding Author: } \\
\hline \multicolumn{2}{|l|}{$\begin{array}{l}\text { Rahul M Patil, } \\
\text { Department of Computer Science and E } \\
\text { R.V. College of Engineering, } \\
\text { Bangalore-560059, Karnataka, India. } \\
\text { Email: rahulpatil61197@gmail.com }\end{array}$} \\
\hline
\end{tabular}

\section{INTRODUCTION}

Internet of Things (IoT) is the new face of technology today. Every application tends to integrate data with its surroundings and this rapid growth of data is responsible for fields like Big Data which enables us to find patterns. With the advent of the IoT, we gather enormous amount of data in real time and this has been driving many technologies we see today.

In this paper, solution proposed is for the problem of finding parking spaces which alle-viates the need to manually search for one, and also significantly reduces the traffic, emission of harmful gases and wastage of fuel which happens as a result of doing so. Time is utilized in searching for a vacant parking space which consumes fuel unnecessarily [1]. Several autonomous parking techniques [2] have surfaced to utilize the available parking space to the maximum extent. Therefore, there is a need to search for a better, smarter and greener solution to this problem. The solution must be composed of sensors to get information about the parking slots, micro-controllers to extract and process this information and modes of communication between micro-controllers and centralized systems to make final decisions based on the final information.

Multiple solutions exist to the problem of finding parking space occupancy. They are broadly classified into Vision-Based approaches and Sensor-Based approaches. Enumerating various issues regarding Image processing techniques such as cost, intractability, the method selected is a sensor-based approach using ultrasonic sensors as it is not just low-cost, but also a highly reliable method to detect parking slot occupancy. For communication between micro-controllers and external devices that represent the users, bluetooth has been utilised for the simple reason that it is low cost and easy to install and implement. 
The system proposed is versatile in the sense that it can easily be replaced with the more popular wireless communication protocols such as Wi-Fi.

An Android application has been developed to connect users to the parking slots by aiding in the process of reserving parking slots remotely. The application uses the Google Maps API to display the map and the current user location, as well as to handle issues in which a person can illegally park in a space reserved by another user. Also, a PaaS, i.e. Firebase has been utilised to store relevant information of each user such as their personal information, payment information and parking space reservation history.

\section{RELATED WORK}

Smart Parking Systems (SPS) have a huge potential in terms of growth and capital re-turns. The commercially active companies in the global market offer a wide range of solutions. Smart Parking Limited based in Scotland engage in designing and maintaining on-street and off-street parking spaces costeffectively. Their solutions include SmartEye sensors, SmartRep management tool, parking map and parking assistance. Omnitec Systems offer solutions such as communication, position of the vehicle, zone controllers and online payment deduction. These are some of the leaders in the global world today and promote a healthy growth towards a sustained environment.

Many problems arise due to double parking of vehicles. In [3] authors have proposed an automated double parking identification and alert system using Internet of Things and Embedded Vision systems. Images are captured and processed on Beaglebone Black (BBB) processor and Region of Interest (ROI) is analysed for violations.

Many research based projects about sensor-based solutions have surfaced recently. The author in [4] presents a paper surveying the different technological breakthroughs that have led to the development of SPS. A concise and succinct comparison is presented to show the advantages and disadvantages of each system. They have proposed a multi-agent parking system based on expert systems, which gains popularity as the trending paradigm for design and conceptualizstion.

In [5] the author has structured his work into 3 layers, a sensor network, a server and a mobile application. The nodes in the sensor layer are of two types, one to detect the stimulus and the other to relay the information to the server. This enhances the system performance by reducing the overload on any given node.

In [6] the author has implemented a smart parking system using internet of things. He has utilized a cloud-based service to store the status of the parking spaces and also the infor-mation about each user. A smart parking guidance system is proposed in [7] using the ZigBee communication protocol to communicate parking lot sensor information to the controller.

The parking assignment problem is still an active area of research. The author in [8] proposed ROSAP, a reservation based smart parking system that uses simulated annealing as a meta-heuristic in order to solve the proposed parking assignment problem formulated as an Integer Linear Program.

\section{METHOD}

The system proposed uses the Arduino Uno to gather sensor data as well as commu-nicate with external devices and modules. Each Arduino is interfaced with an HC-05 Bluetooth module to communicate with our Android devices. These HC- 05 modules are used to represent the Arduinos as being nodes in an interconnected network. Each of the nodes are interfaced with HC-SR04 ultrasonic sensors that are used to detect the occupancy of the parking slots by using ultrasonic signals to detect distance to objects.

Each of the parking slots are also equipped with RGB LEDs to indicate whether a parking slot is empty, occupied or reserved with emission of colour like green, red and blue respectively. These LEDs are lit according to either the occupancy of a parking slot that is determined by the values coming in from the sensor or in case of reservation, it may depend on whether a user of the Android app has reserved the slot in advance. The reservation of a certain parking slot is communicated via the Bluetooth module.

\subsection{Controller Area Network (CAN)}

Controller Area Network also known as the CAN Bus is a fast, low cost and sturdy message-based protocol that allows micro-controllers to communicate with each other without the aid of a host device. It is generally used in the automotive industry to connect the various subsystems within the vehicle. It also finds great use in short range communication between micro-controllers for embedded systems as proposed in this paper.

CAN networks are useful in applications where there is need for small amounts of data to be shared over a network at regular intervals used very similarly in a Smart Parking System as in [10]. The protocol 
used in CAN is the CSMA/CD+AMP type [9], which means that it is a carrier-sense, multiple-access protocol with collision detection and implementation of message priority using bit-wise arbitration.

In the network, each of the frames have a unique identifier or a frame ID that is used to determine where its coming from and what type of data it is carrying along with it.

\begin{tabular}{|c|c|c|c|c|c|c|c|c|c|c|}
\hline $\begin{array}{c}\text { Start } \\
\text { of } \\
\text { Frame }\end{array}$ & $\begin{array}{l}\text { 11-bit } \\
\text { Identifier }\end{array}$ & $\begin{array}{l}\mathbf{R} \\
\mathbf{T} \\
\mathbf{R}\end{array}$ & $\begin{array}{l}\text { I } \\
\text { D } \\
\text { E }\end{array}$ & RO & $\begin{array}{l}\text { D } \\
\text { L } \\
\text { C }\end{array}$ & $\begin{array}{l}\text { Payload } \\
\text { (0-8 bytes) }\end{array}$ & $\begin{array}{l}C \\
R \\
C\end{array}$ & $\begin{array}{l}\mathrm{A} \\
\mathrm{C} \\
\mathrm{K}\end{array}$ & $\begin{array}{l}\text { End of } \\
\text { Frame }\end{array}$ & $\begin{array}{l}\text { I } \\
F \\
S\end{array}$ \\
\hline
\end{tabular}

Figure 1. Format of standard CAN Frame

The above Figure 1illustrates the frame format used in the Standard CAN which is used in the SPS proposed. The payload of the frame has a maximum size of 8 bytes, and uses an 11-bit identifier that aids in defining the priority of the message being sent. 4-bit DLC contains the length of the message being transmitted. The CRC is a 16-bit checksum that is used for error detection and ACK is used as an acknowledgement for transmission of error free messages. RTR is a single remote transmission request that is high to indicate that information is required from another node. IDE is the identifier extension that represents whether the identifier is being transmitted with or without extension. The 7-bit IFS or inter-frame space defines the amount of time the controller may take to transfer received message to the message buffer successfully.

\subsection{Client-Side Application}

An Android application has been developed which is connected to the server to receive data about empty slots in the user's vicinity. The application is also running the Google Maps API for Android which gives the user's current location and the location of available parking slots in his vicinity. The time of entry and time of exit is calculated automatically and a bill is generated based on factors like time, duration, and demand.

We have used a Bluetooth module (HC-05), which is a low Bluetooth energy device that simulates the wireless communication between the user and the sensor system deployed at the parking spaces. The user selects a slot of his convenience and the Google Maps API is used to generate the shortest path to guide the user. The interface has been made such that the usage is quite simple along with providing all the above functionalities.

For the purpose of simulation, we have used the Firebase SDK compatible with Android. We have used NodeJS to run the backend scripts that queries information stored on cloud. We have also used FCM which is the cloud message service provided by Firebase to send push notifications to the user for relaying the payment details. It is also used for authentication of each user via simple login as shown in Figure 2.

\subsection{Design}

In the system proposed, the CAN protocol has been used as secondary source of commu-nication in case of failure in the wireless communication that has been achieved using Bluetooth. Arduinos are interfaced with the CAN controller MCP2515 and high-speed transceivers named MCP2551.

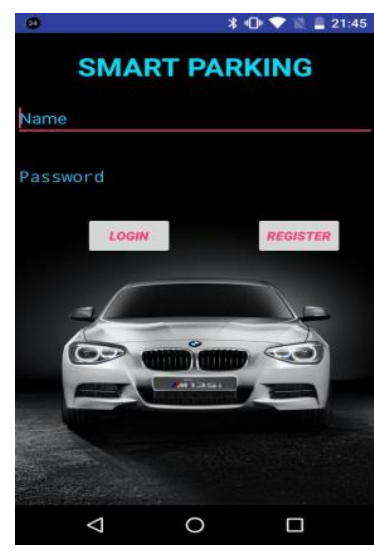

Figure 2. Login activity of android app

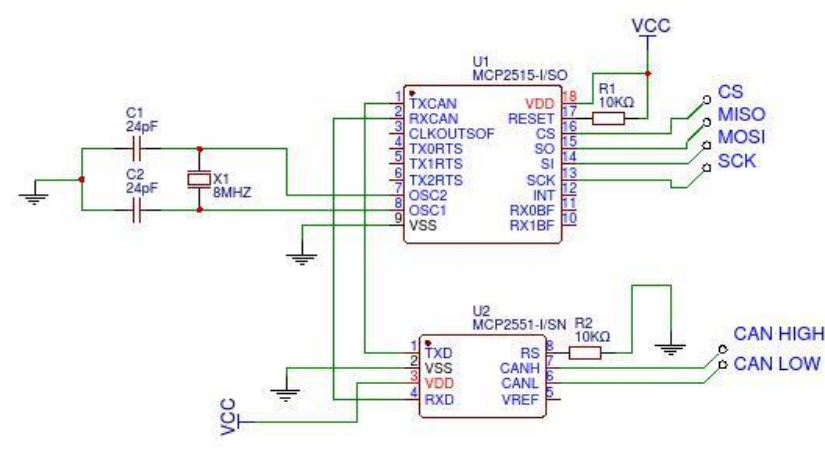

Figure 3. CAN controller and high-speed transceiver 
These integrated chips facilitate the communication between Arduinos using the stan-dard CAN protocol. The micro-controllers are connected in a bus topology, each transmitting the required bytes of information regarding information of the parking slots to every other micro-controller on the CAN Bus.

The distinction in the frame identifiers is what aids in identification of which exact parking slots a certain byte of data pertains to. This is an essential addition to the Smart Parking System that has been proposed, to act as a second line of communication in case of failure with the wireless communication that may have already been used in the system.

\section{EXPERIMENTAL RESULTS}

The system has been tested successfully using Arduino Unos interfaced with ultrasonic sensors, with each sensor identifying as a single parking slot. The circuit implementation of the system is as shown in Figure 5.

When given a power supply, the circuit begins to function, and the sensors begin to sense:

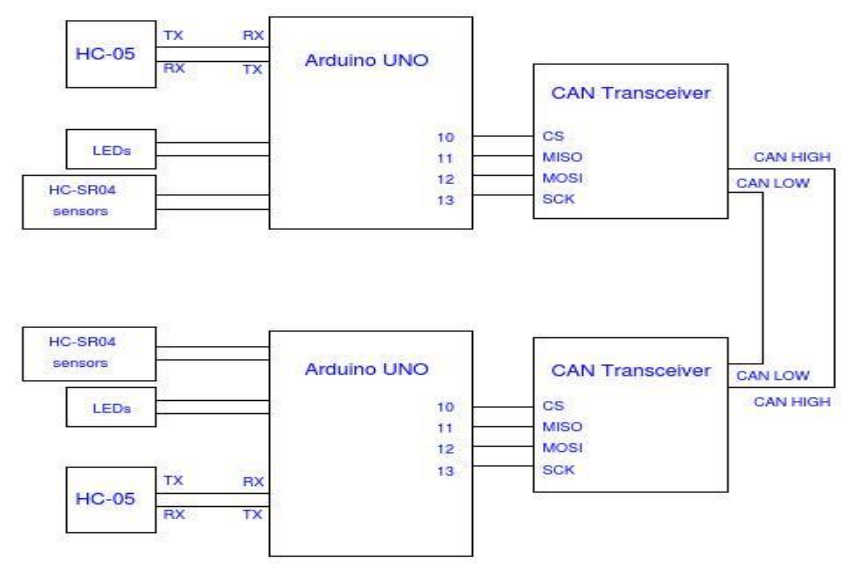

Figure 4. Block representation of circuit with can bus

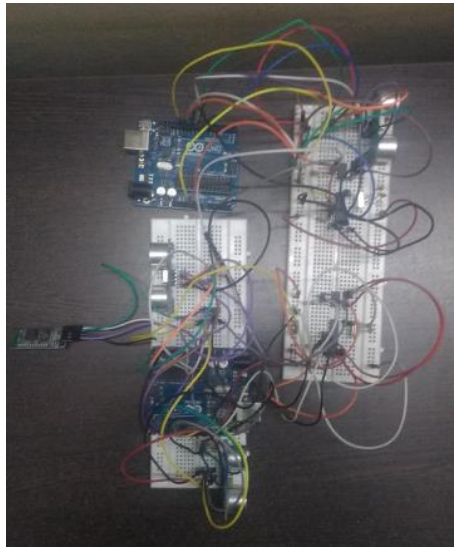

Figure 5. Circuit Setup

The vacancy of parking spots continuously and simultaneously sends it both, wirelessly as well as broadcast on the CAN Bus. The green colour of the LEDs in Figure 6 (a) represents the vacancy of the parking slots, and the same information has been transmitted to the Android app as shown in Figure 6 (c) where it is clearly shown that all slots are vacant and open to reservation.

Identically, the same can be observed in the app, represented by the green colour of the corresponding parking slot reservation buttons. Now, considering that a certain car has come and occupied a certain parking slot, the LED turns red indicating the same and the status of it is transmitted to the Android app as shown in the Figure 6 (b) and Figure 6 (d).

To show reservation of a certain parking slot, we simply blink the LED in the red colour. This conveys to other motorists who are manually searching for parking slots that the slot has been reserved by a user. The issue of prevention of illegal parking of the pre-reserved parking slots by motorists has been addressed using physical barriers and the maps API. A physical barrier such as spikes or gates can be used to prevent illegal parking. The maps API comes into play to disable this physical barrier when the rightful user of the parking slot arrives. When the registered user enters in a certain pre-defined region of the parking slot, then the physical barrier gets disabled and allows to user to conveniently utilise the parking space. Simultaneously the user is also charged for the service utilised using the payment details and other relevant information as stored in the real-time database in the cloud services provided by Firebase.

In case of failure of the wireless transmission between the micro-controllers and the user's devices, the CAN Bus proposed comes to the aid of the system, where frames are sent by each micro-controller denoting information about the parking slots connected to it. Below is a repre-sentation of the CAN frame data as received by the Arduino on the serial monitor. A single frame 


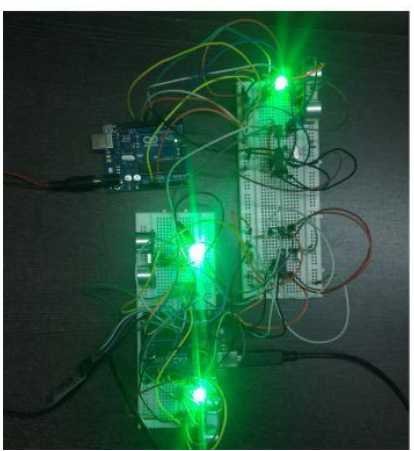

(a) All parking slots are vacant

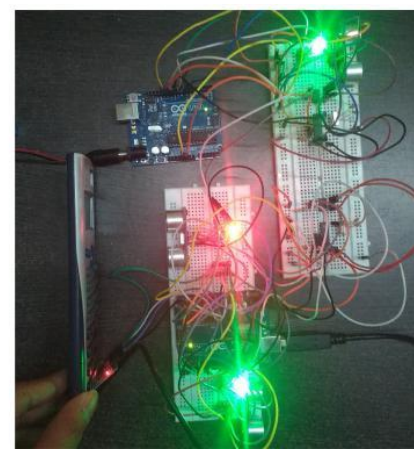

(b) Single slot occupied

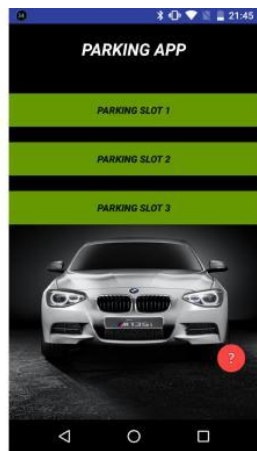

(c) Android app de picting vacancies

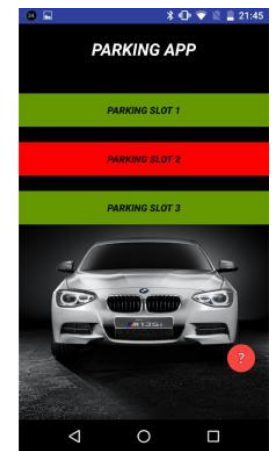

(d) Android app depicting occupancy

Figure 6. Application-based smart parking system

Received with a hexadecimal ID of ABBA is shown to be received with each byte holding information about the parking slots interfaced by the micro- controller sending that particular frame of data.

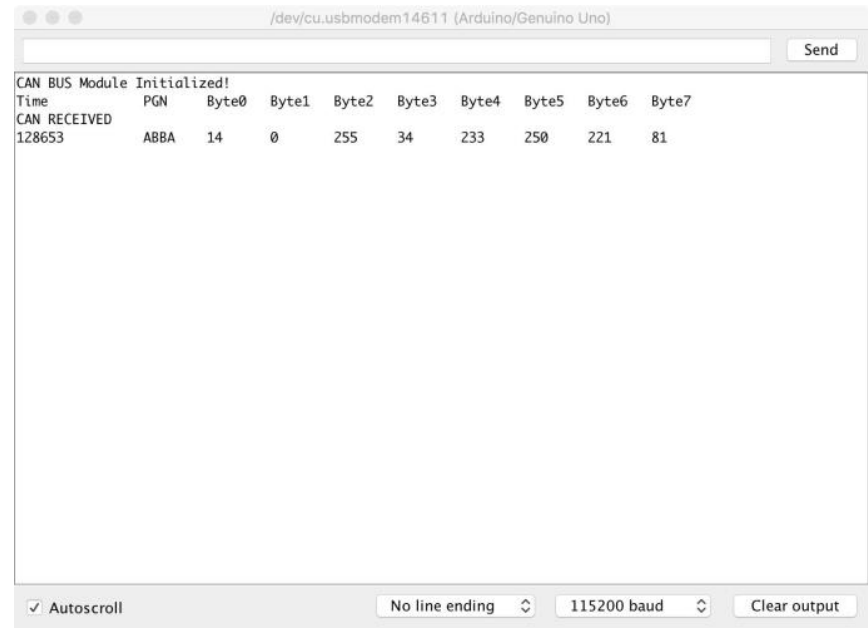

Figure 7. Circuit Setup

When this failure occurs, the node gets cut off from the wireless communication and its information regarding the parking slot statuses is lost. Here, the CAN Bus connecting the micro-controllers comes into action, where the damaged node has its information conveyed through another functioning micro-controller connected to the same CAN bus. This plays an essential role as it prevents parking slots from going undetected and unused.

\section{CONCLUSION}

Smart Cities are the future of tomorrow all around the world and various systems that co-exist within these environments make the city smart. One such system is the Smart Parking System, and the system proposed in this paper greatly automates the process by directly con-necting the parking areas to the civilians by providing a platform in the form of an application. This application enables them to reserve these parking slots in advance and reduces the need of manually searching for them. This significantly reduces fuel usage and as a result of which reduces pollution in the city. To address to problem of node failure within a system such as this, that could cause several parking slots to go undetected or unused, the CAN protocol has been utilised. Therefore, the solution to parking proposed here is connected, robust and green which are the main requirements of any Smart system. 


\section{FUTURE WORK}

In any Internet of Things project, reliability and sustainability are two major factors that decide its effectiveness. Increasing the number of sensor nodes and parking spaces will give us a broader view of tackling situations that arise in real-time. Considering factors like user history, demand of a location, reviews about a location, we can deliver relevant information about the place to our users. In general, we will aim to focus more on the sustained use of resources available and deliver a better user experience.

\section{REFERENCES}

[1] Himal Pratap Singh, et al., "An Approach to Implement Cost Efficient Space Detection Technol-ogy with Lower Complexity for Smart Parking System," in TELKOMNIKA Indonesian Journal of Electrical Engineering, DOI:10.11591/telkomnika.v15i3.8456, 2015.

[2] Zheng Guoqiang, et al., "Optimization of an Intelligent Controller for Parallel Autonomous Park-ing," in TELKOMNIKA Indonesian Journal of Electrical Engineering, ISSN: 2302-4046, 2013.

[3] Noryasikin Fadilah, et al., "Embedded Automated Vision for Double Parking Identification Sys-tem," in Indonesian Journal of Electrical Engineering and Computer Science, ISSN: 2502-4752, DOI: 10.11591/ijeecs.v10.i3.pp1221-1226, 2018.

[4] Khaoula Hassoune, et al., "Smart Parking Systems: A Survey," in IEEE ISBN:978-1- 5090-5781-8/16, 2016.

[5] H.M.A.P.K Bandara, et al., "Smart Campus Phase One: Smart Parking Network," Proceedings of the 1st Manufacturing and Industrial Engineering Symposium., Department of Computer Engineering, University of Peradeniya, 2016.

[6] Basavaraju S R., "Automatic Smart Parking System using Internet of Things(IoT)”, Interna-tional Journal of Scientific and Research Publications, Volume 5, Issue 12, December 2015.

[7] Wenyu Cai, et al., "Implementation of Smart Parking Guidance System based on Parking Lot Sensors Networks," at Proceedings of ICCT, 2015.

[8] Naourez Mejri, et al., "Reservation based multi-objective Smart Parking approach for Smart Cities," in IEEE, ISBN:978-1-5090-1846-8/16, 2016.

[9] Steve Corrigan, "Introduction to the Controller Area Network (CAN)," in Application report SLOA101B, Texas Instruments, 2016.

[10] Jian Wan, et al., "Design and verification of CAN bus based devices for parking spaces detection," in Proceedings of 2014 IEEE International Conference on Service Operations and Logistics, and Informatics, DOI: 10.1109/SOLI.2014.6960691, 2014. 